\title{
Modelling and PID control system integration for quadcopter DJIF450 attitude stabilization
}

\author{
N. M. Salma, Khairuddin Osman \\ Advanced Sensors and Embedded Control Systems (ASECs), \\ Centre for Telecommunication Research and Innovation (CeTRI), Faculty of Electronics and Computer Engineering,
} Universiti Teknikal Malaysia Melaka, Malaysia

\begin{tabular}{l} 
Article Info \\
\hline Article history: \\
Received Feb 1, 2020 \\
Revised Mar 16, 2020 \\
Accepted Apr 7, 2020 \\
\hline
\end{tabular}

Keywords:

DJI F450

Mathematical model

Newton-euler

UAV

\begin{abstract}
In this paper we focus on the overall overview of the mathematical modelling of the DJI F450 UAV quadcopter, the hardware and software system integration based on PID control system for the attitude feedback. The parameter specification of the DJI F450 is fed into the mathematical model and implement a basic PID for the system. Future research using the DJI F450 model can benefit from this observation by implementing the modelling and tune in their own variable that varies, such as the overall of their weight. The data of PID control system simulation using the quadcopter frame model type DJI F450 parameter. The mathematical model for the quadcopter modelled DJI F450 is developed using Newton-Euler method. Altitude data for the control system is obtain from the analysis data of the Simulink simulation. The simulation is done using the Simulink toolbox inside the MATLAB software. From this paper, we can more understand the step involves in making a full control system of a quadcopter. The mathematical model for other type of quadcopter model can be implemented using the steps with their own parameter and achieve fast development.
\end{abstract}

Copyright $@ 2020$ Institute of Advanced Engineering and Science. All rights reserved.

\section{Corresponding Author:}

Khairuddin Osman,

Faculty of Electronics and Computer Engineering, Universiti Teknikal Malaysia Melaka,

Hang Tuah Jaya, 76100 Durian Tunggal, Melaka, Malaysia.

Email: khairuddin.osman@utem.edu.my

\section{INTRODUCTION}

Quadcopter is considered in the Multirotor Vertical Take-off and Landing (VTOL) type Unmanned Aerial Vehicle (UAV). The VTOL capability is that it can handle Conventional Take-off and Landing (CTOL), whereby it will be easy for the quadcopter to fly off the ground with a small surrounding space, and able to fly through small, and narrow path. UAV also known as Unpiloted Aerial Vehicle competent to fly without any human pilot onboard. The technology is favored to use in the military department mostly surveillance in remote, and dangerous places where it can save valuable human recourses. There is various version of quadcopter that had been developed found in research literature such as micro or nano sized quadcopter usually used in swarm and surveillance [1-3], aerial cinematography type, where the quadcopter able to lift a specific camera $[4,5]$. Moreover, there are also sport FPV H-type frame quadcopter where it specialized in racing $[6,7]$.

The modelling behavior of the quadrotor is premeditated in this work and focusing on improving the take-off stability and overall flight performance. The quadcopter has a lot of advantages compared with other types of aircraft, whereby it has good maneuverability, simple assembly and it have a great room of improvements and innovation that can be done with the quadcopter itself. Basic component of a quadcopter consists of four brushless motors and its propellers, a battery to power the quadcopter, embedded processor, and sensors. The challenge in the project is how to design the controller of the quadrotor system to obtain 
stability and achieve the desired state when there are many uncertainties in the environment system, for example, external disturbance, motor actuation degradation, time delays [8], and also sensor error [9]. The project focuses on modelling the DJI F450 quadcopter, and design the specific controller using PID. There are many types of control system method in controlling the altitude of the quadcopter based on these research [6-12]. The mathematical model of the quadcopter is derived from previous research and the DJI F450 frame parameter was embed into the model. The motivation for this research is to ease the DJIF450 user for embedding the control system used for faster development of their application using the quadrotor.

\section{MATHEMATICAL MODELLING}

The mathematical model for the quadcopter is essential to be determined first for rapid analysis of the simulation result from the hardware. For this paper, the basic mathematical model of a quadcopter was implemented and implement with the DJI F450 frame specs. Studies by [12-17] was heavily referred for the modelling quadcopter UAV of this paper. The quadcopter model itself is a common symmetrical frame that have four arms. Each motor is assigned at the far end of the arms while the ESC was placed beneath the middle of the arm. The battery of the quadcopter is placed on the center of the quadcopter as in a common quadcopter setup [9]. The custom power distribution PCB and custom controller was embedded on the center of the frame.

\subsection{Basic Concepts}

There are two possible configurations for most of quadcopter designs " + " and " $x$ " as shown in Figure 1. An " $x$ " configuration quadcopter is considered to be more stable compared to "+" configuration, which is a more acrobatic configuration. Propellers 1 and 3 rotates clockwise $(\mathrm{CW}), 2$ and 4 rotates counter clockwise $(\mathrm{CCW})$. According to the Third Newton Law, every force applied there will be an equal feedback force will be act to the same object, thus in the case of the quadcopter the force of the rotors pushing air downwards, the same force feedback will make the quadcopter itself to move the opposite way of the air pushed by the rotors, and projection achieved.

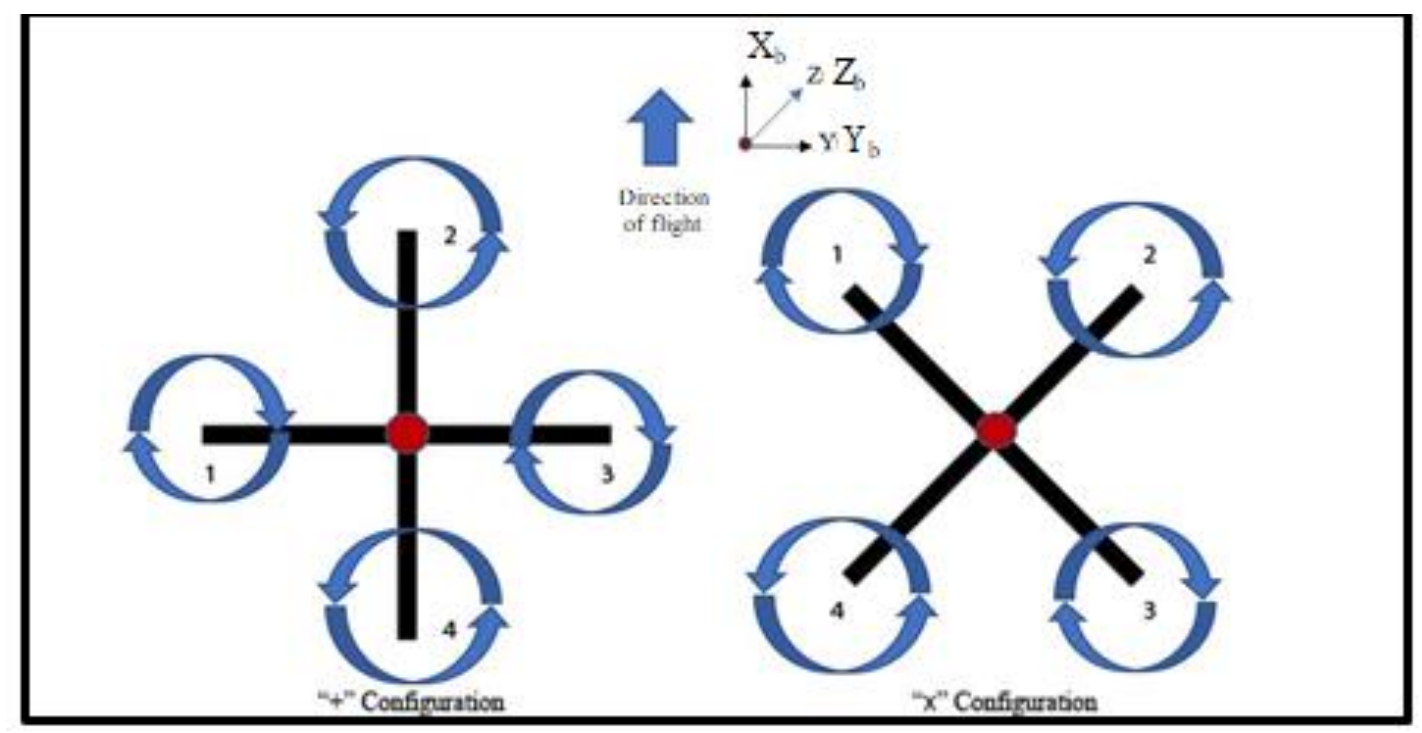

Figure 1. "+" and " $\mathrm{x}$ " configuration for the quadcopter

Propellers 1 and 3 have same length to 2 and 4, so all thrusts have the same direction [18]. When all four motors are spinning at the same speed, the rotors create equal thrust that lifts the quadrotor into the air. The constant action for all of the component indicate that the quadcopter is in hovering mode, where the attitude of the quadcopter remains the same. To change the pitch attitude for the "+" configuration, the speed of motor 1 is reduced while the speed of motor 3 is increased, or vice versa, creating a non-zero pitch angle.

As both motor 1 and motor 3 are rotating in the same direction the total counteracting torque provided is not changed so the quadrotor maintains its yaw angle. The roll attitude is adjusted in a similar 
manner. To adjust the yaw angle, the speed of motors 1 and 3 are increased while the speed of motors 2 and 4 are decreased, or vice versa. This creates an imbalance in the total torque in the yaw axis and so the quadrotor will change yaw angle [19].

\subsection{Motion of Quadcopter}

The most intuitive way of understanding the motion of a quadrotor is by investigating how the rotation of the individual rotors affect the motion of the body. Equal speed on all rotors, resulting in equal forces from each rotor. This allows the quadrotor to move along the $\mathrm{z}_{\mathrm{b}}$ axis by increasing or decreasing the rotor speed. With a certain rotor speed the quadrotor will obtain a hovering state. In both cases two adjacent rotors have increased speed compared to the other rotors, resulting in a movement around the $\mathrm{x}_{\mathrm{b}}$ and $\mathrm{y}_{\mathrm{b}}$ axes respectively. Each rotor affects the quadrotor body with a torque in the opposite direction of the rotation of the rotor. These torques are canceled when two rotors rotate clockwise and two rotors rotate counterclockwise with the same speed. The torque from the rotors can be exploited to control the yaw angle $\psi_{\mathrm{b}}$, where two diagonal rotors rotate faster than the other rotors. This result is a movement around the $\mathrm{z}_{\mathrm{b}}$ axis, in this case in the positive yaw direction. To designate the motion of 6 DOF rigid body it is normal to define with two reference frames [20, 21]. In the Figure 2 shows the coordinate system and the body frames used. The advantage of body symmetry is it can simplify the equations associate with the system [22]. The B and the E symbols represent the body-fixed frame and the earth initial frame respectably. The linear position vector of the earth inertial frame axis $\mathrm{x}, \mathrm{y}, \mathrm{z}$ is defined with $\xi_{E}$ as shown in (1).

$$
\begin{aligned}
& \xi_{E}=\left[\begin{array}{lll}
\mathrm{x} & y & z
\end{array}\right]^{T} \\
& \vartheta_{E}=\left[\begin{array}{lll}
\varphi & \emptyset & \theta
\end{array}\right]^{T} \\
& \mathrm{~V}_{\mathrm{b}}=\left[\begin{array}{lll}
\mathrm{u} & \mathrm{v} & \mathrm{w}
\end{array}\right]^{\mathrm{T}} \\
& \omega_{\mathrm{b}}=\left[\begin{array}{lll}
\dot{\varphi} & \dot{\emptyset} & \dot{\theta}
\end{array}\right]^{\mathrm{T}}
\end{aligned}
$$

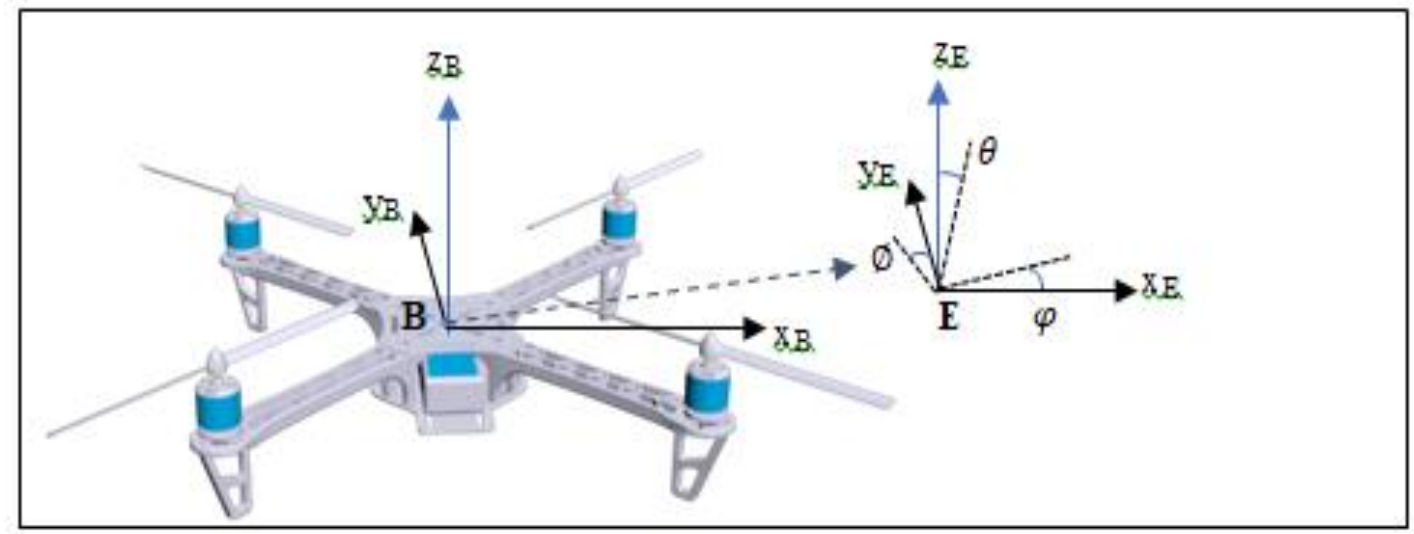

Figure 2. Quadcopter DJI F450 body frames and coordinate system used

Moving on to the (2), $\vartheta_{E}$ is the three Euler angle consist yaw pitch roll, where $\varphi$ is the yaw angle where rotates around the $\mathrm{z}$ axis. The roll angle, $\emptyset$ rotates around the $\mathrm{x}$ axis, and lastly the pitch angle $\theta$ rotates around the $\mathrm{y}$ axis. In (3), $\mathrm{v}_{\mathrm{b}}$ represent the linear velocity which is $\mathrm{u}$ is the velocity in $\mathrm{x}$ axis, $\mathrm{v}$ is the velocity in y axis, and $\mathrm{w}$ is the velocity in the $\mathrm{z}$ axis. Moving on to the (4), $\omega_{\mathrm{b}}$ is the angular velocity where the $\dot{\varphi}$ is the rate of change of yaw, $\dot{\emptyset}$ is the rate of change of roll angle, and lastly the pitch angle is identified by $\dot{\theta}$ in this specific application. The quadrotor dynamical equations used is based on the Newton-Euler formalism, where the full non-linear dynamics can be expressed in combination of gravitational vector, gyroscopic torque vector and movement vector [23]. The summation of force required to accelerate the mass of the quad, $\mathrm{m}_{\mathrm{V}}$ and the centrifugal force of the quad body, $\omega_{b}\left(\mathrm{mV}_{\mathrm{b}}\right)$ is equal with the gravity $\mathrm{gR}^{\mathrm{T}}$ and the total thrust from the rotors $T_{b}$ as shown in the (5). Where $m$ is the mass of the drone, $\dot{V}_{b}$ is the linear acceleration of the fix body drone, $\omega_{b}$ is the angular velocity of the fix body drone, $V_{b}$ is the linear velocity of the fix body drone, $\mathrm{g}$ is the gravity acting on the fix body drone, $\mathrm{R}^{\mathrm{T}}$ is the rotation matrix from body frame to the initial 
frame from (8), and $T_{b}$ is the thrust of the rotors. The angular acceleration of the inertia can be obtain from (6), where $J^{\mathrm{b}}$ is the inertia matrix stated in (12) and $\tau_{\mathrm{b}}$ is the gyroscopic forces acting on the body fixed frame Rotation matrix from the body frame to the inertial frame is stated in (7) and (8) [24].

$$
\begin{aligned}
& \mathrm{m}_{\mathrm{b}} \times \omega_{\mathrm{b}}\left(\mathrm{mV}_{\mathrm{b}}\right)=\mathrm{gR}^{\mathrm{T}}+\mathrm{T}_{\mathrm{b}} \\
& J^{\mathrm{b}} \omega_{\mathrm{b}}=-\left(\omega_{\mathrm{b}} \times J^{\mathrm{b}} \omega_{\mathrm{b}}\right)+\mathrm{T}_{\mathrm{b}}-\tau_{\mathrm{b}} \\
& \mathrm{R}=\mathrm{R}(\emptyset, \mathrm{z}) * \mathrm{R}(\theta, \mathrm{y}) * \mathrm{R}(\varphi, \mathrm{x}) \\
& R=\left[\begin{array}{ccc}
\cos \theta \cos \varphi & \sin \emptyset \sin \theta \cos \varphi-\cos \emptyset \sin \varphi & \cos \emptyset \sin \theta \cos \varphi+\sin \emptyset \sin \varphi \\
\cos \theta \sin \varphi & \sin \emptyset \sin \theta \cos \varphi+\cos \emptyset \sin \varphi & \cos \emptyset \sin \theta \sin \varphi-\sin \emptyset \cos \varphi \\
-\sin \theta & \sin \emptyset \cos \theta & \cos \emptyset \cos \theta
\end{array}\right] \mathrm{b}
\end{aligned}
$$

Rotation matrix $\mathrm{R}$ is orthogonal, thus $\mathrm{R}^{\urcorner 1}$ is equal to $\mathrm{R}$ which is the rotation matrix from the inertial frame to the body frame. There are 3 types of angular speeds which can describe as the derivative of $(\varnothing, \theta, \psi)$ with respect to time, that are $\varnothing$ is the roll rate, $\theta$ is the Pitch rate, and $\psi$ is the Yaw rate. Considering the hovering condition of quadcopter gives 4 equations of forces, directions, moments and rotation speeds. By increasing or decreasing the rotation speed of all the propellers, the quadcopter can make movements flying up and down.

\subsection{Equation of motion}

For the modelling simulation, the equation had been put on the MATLAB and Simulink software, while the vital parameter that need to be used in the model was identified. On the Figure 3 shows the forces and moments parameters acting on a rotor. The resultant force acting on the blade's elements are perpendicular to the rotor shaft. The thrust force parameter is represented in (9). The thrust generated from the motors and the propellers drives the quadcopter to the desired state.

$$
\begin{aligned}
& \mathrm{T}=\mathrm{C}_{\mathrm{T}} \rho \mathrm{A}(\omega \mathrm{R})^{2} \\
& \mathrm{M}_{\mathrm{Q}}=\mathrm{C}_{\mathrm{MQ}} \rho \mathrm{A}(\omega \mathrm{R})^{2} \mathrm{R} \\
& \mathrm{M}_{\mathrm{R}}=\mathrm{C}_{\mathrm{MR}} \rho \mathrm{A}(\omega \mathrm{R})^{2} \mathrm{R}
\end{aligned}
$$

$\mathrm{T}$ is the thrust equation used for the motors, where specifically $\mathrm{T}$ is the thrust force. $\mathrm{C}_{\mathrm{T}}$ is the thrust coefficient that is related directly to the aerodynamic efficiency of the rotor, while indirectly will show how much the propeller affects air flow and thrust by extension. $\rho$ is the density of air, A is the cross section area of the propeller's rotation, $\omega$ is the angular velocity of the rotor and $\mathrm{R}$ is the radius of the rotor. Torque is the resultant moment of all horizontal forces acting to the center of the rotor that determines the power required from the motor to keep the quadcopter fly, represented by the drag moment $\mathrm{M}_{\mathrm{Q}}$ and the rolling moment in the quadcopter is assume as $\mathrm{M}_{\mathrm{R}}$.

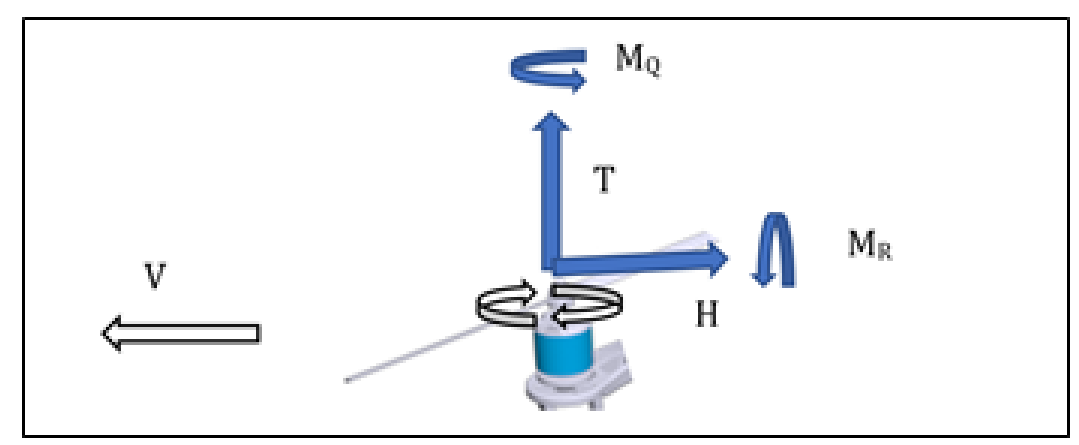

Figure 3. Forces acting on a rotor [17] 


\subsection{Compiled Mathematical Model}

The simplified mathematical model has been developed based on the basic mathematical model framework that have reviewed in the understanding the range of variable involved in modelling the UAV. The method of simulation using MATLAB and Simulink using the opensource tool provide a slightly simplified mathematical model.

$$
J^{\mathrm{b}}=\left[\begin{array}{ccc}
J x & 0 & 0 \\
0 & J y & 0 \\
0 & 0 & J z
\end{array}\right]
$$

In (12) is the translational motion for describing the mass is one of the vital parameters for the quadcopter rotational motion that required to mathematically describe it as moment of inertia, $J^{\mathrm{b}}$. Two methods that are usually used to determine the inertia parameter which are the moment of inertia calculation and by bifilar pendulum experiment. The initialization of the equation consists of parameter of the motors, the electronic speed controller unit (ESC), the central frame, and the arms used in the quadcopter. Thus, the total moment of inertia equation for the DJI 450 quadcopter is in (13).

$$
\mathrm{J}_{\mathrm{i}}=\mathrm{J}_{\mathrm{i}, \mathrm{M}}+\mathrm{J}_{\mathrm{i}, \mathrm{S}}+\mathrm{J}_{\mathrm{i}, \mathrm{H}}+\mathrm{J}_{\mathrm{i}, \mathrm{A}} \text { for } \mathrm{i}=\mathrm{x}, \mathrm{y}, \mathrm{Z}
$$

In (14) shows the relationship between the thrust, motors angular velocity, thrust coefficient, and the length of the arm of the frame, where $d_{x}$ is arm length of the DJI F540 frame. To simplify the equation, $\rho \mathrm{A}_{\mathrm{r}} \mathrm{r}^{2}$ is combined into $\mathrm{C}_{\mathrm{T}}$, thus obtain the thrust coefficient relation. The torque force of the motor system is $\mathrm{Q}=\mathrm{C}_{\mathrm{q}} \omega^{2}$ where $\mathrm{C}_{\mathrm{q}}$ includes $\rho \mathrm{A}_{\mathrm{r}} \mathrm{r}^{2}$ parameters. From all of the equations, the matrix which describes the thrusts and torques on the system can now be generated. The percent throttle is converted into RPM using the linear regression method in (15). Where $\tilde{\omega}_{\mathrm{ss}}$ is the expected steady state motor RPM, Throttle $\%$ is the command input by the user in percentage value. $\mathrm{C}_{\mathrm{r}}$ is the throttle command values from percentage to RPM conversion coefficient. $b$ is the $y$-intercept of the linear regression relationship.

$$
\begin{aligned}
& {\left[\begin{array}{c}
\sum T \\
T_{\varnothing} \\
T_{\theta} \\
T_{\varphi}
\end{array}\right]=\left[\begin{array}{cccc}
\mathrm{C}_{\mathrm{T}} & \mathrm{C}_{\mathrm{T}} & \mathrm{C}_{\mathrm{T}} & \mathrm{C}_{\mathrm{T}} \\
-d_{x} \mathrm{C}_{\mathrm{T}} & -d_{x} \mathrm{C}_{\mathrm{T}} & d_{x} \mathrm{C}_{\mathrm{T}} & -d_{x} \mathrm{C}_{\mathrm{T}} \\
-d_{x} \mathrm{C}_{\mathrm{T}} & -d_{x} \mathrm{C}_{\mathrm{T}} & d_{x} \mathrm{C}_{\mathrm{T}} & d_{x} \mathrm{C}_{\mathrm{T}} \\
-C_{q} & C_{q} & -C_{q} & C_{q}
\end{array}\right]\left[\begin{array}{c}
\omega_{1}^{2} \\
\omega_{2}^{2} \\
\omega_{3}^{2} \\
\omega_{4}^{2}
\end{array}\right]} \\
& \tilde{\omega}_{\mathrm{ss}}=\left(\text { Throttle } \% * \mathrm{C}_{\mathrm{r}}\right)+\mathrm{b}
\end{aligned}
$$

\section{SIMULATION AND ANALYSIS}

The obtained physical parameter data for the quadcopter DJI F450 that is used is tabulated in Table 1, the values were applied in the simulation to get as close to the physical data for the hardware quadcopter of the same model. Where, $\mathrm{dm}$ is distance from motor to center frame, ESC width is the distance

\begin{tabular}{|c|c|c|c|}
\hline Motors & ESC & Central Frame & Arms \\
\hline $\begin{array}{l}\text { Mass }=48 \mathrm{~g} \\
\mathrm{dm}=30 \mathrm{~cm}\end{array}$ & $\begin{array}{c}\text { Mass }=25 \mathrm{~g} \\
\text { width }=2.4 \mathrm{~cm}\end{array}$ & $\begin{aligned} \text { Mass } & =70 \mathrm{~g} \\
\text { radius } & =6 \mathrm{~cm}\end{aligned}$ & $\begin{array}{l}\text { Mass }=55 \mathrm{~g} \\
\text { radius }=2 \mathrm{~cm}\end{array}$ \\
\hline $\begin{array}{l}\text { Height }=2.75 \mathrm{~cm} \\
\text { radius }=1.35 \mathrm{~cm}\end{array}$ & $\begin{array}{c}\text { length }=4.5 \mathrm{~cm} \\
\mathrm{ds}=6.0 \mathrm{~cm}\end{array}$ & Height $=4.29 \mathrm{~cm}$ & Length $=18.5 \mathrm{~cm}$ \\
\hline 3s Lipo battery mass & & & $404 \mathrm{~g}$ \\
\hline Torque coefficient: $\mathrm{C}_{\mathrm{q}}$ & & & $7 \mathrm{Nm} / \mathrm{RPM}^{\wedge} 2$ \\
\hline Thrust coefficient: $C_{t}$ & & & $-6 \mathrm{~N} / \mathrm{RPM}^{\wedge} 2$ \\
\hline Throttle $\%$ to RPM: $\mathrm{C}_{\mathrm{r}}$ & & & 46.48 \\
\hline Motor $\mathrm{kV}$ rating & & & $1000 \mathrm{kV}$ \\
\hline
\end{tabular}
ESC to central frame. The length of the components was measured using a standard ruler and the mass of the components were measured using a digital scale.

Table 1. Physical parameter

Figure 4 shows the quadcopter used on taking the parameter to be implement on the simulation. The frame of the quadcopter used is using the DJI F450 model frame. The top hub frame had been modified into an embedded pcb board consists of the microcontroller, sensor and communication dongle, in this case, we use rf transceiver chip. 


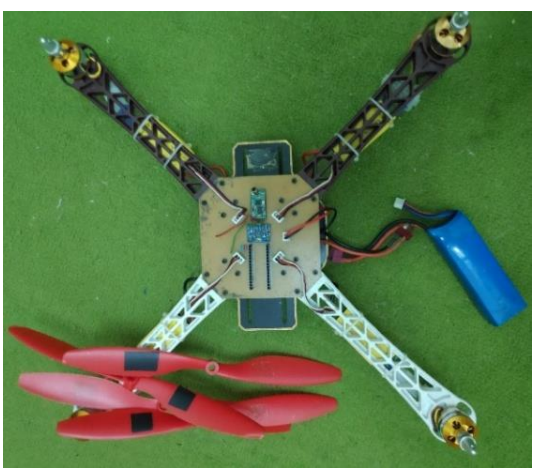

Figure 4. The quadcopter parameter used

\subsection{Basic Closed Loop Simulation}

For the closed loop simulation, the desired attitude command will be sent to the quadcopter using the parameters for the specific DJI450 quadcopter frame that is currently using. In Figure 5 shows the overall complete embed control system used. The control system block begins with the attitude command block, where we set the initial position of the quad at the starting step time, and set the desired position at the end of the step time. The attitude command signal is then feed into the PID controller. Based on (16), basic PID was used in the PID block. The PID gains were tune manually. The integral component for this model was not used because of the system lacking negative value reading. The PID tuning was first started in the Z, or the altitude component, because it's easier to let the quadcopter hover steady before moving on to the other attitude components of the quadcopter.

$$
u(t)=K_{P} e(t)+K_{i} e(t)+K_{d}\left(\frac{d}{d t}\right) e(t)
$$

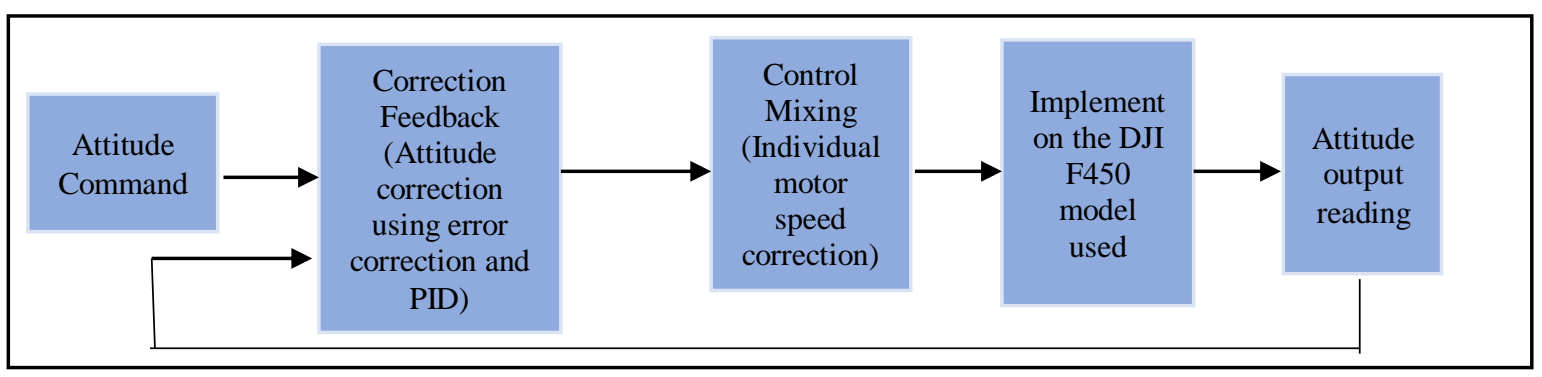

Figure 5. The overview of the control system block

After going on the PID block, the data then is proceeded on the next block which is the mixing block. The mixing block will determine which motor will be turn with the correct speed to get the desired position of the quadcopter. Moving on to the next block, the mathematical parameter from earlier will be used on the mathematical modelling. The inertia, disturbance, drag force and all of the parameter will determine the true position of the quadcopter. The output from this block, was observed, and the output also will be the input feedback for the PID in the previous PID controller block.

Figure 6 shows the screenshot for the control system used in the simulation of the quadcopter using physical parameter model DJI F450. Figure 7 until Figure 10 shows the analysis result for the specific desired command defined for the program initialization. For the first three figure, the phi, theta and the psi position result shows that the correction happens before the desired time step, this is because in the PID controlling block, an auto correction system for the quadcopter was embedded, thus useful for auto hovering and stabilizing of the quadcopter $[6,14,19,25]$. In the altitude position result simulation, the initial position of the quadcopter was at 3 , and it follows the initial desired position which is 1 , and swiftly correcting itself. When the time step is after 10 , the desired position of the altitude is 8 , the quadcopter manages to maneuver to the desired $\mathrm{Z}$ or altitude position within a short time. 


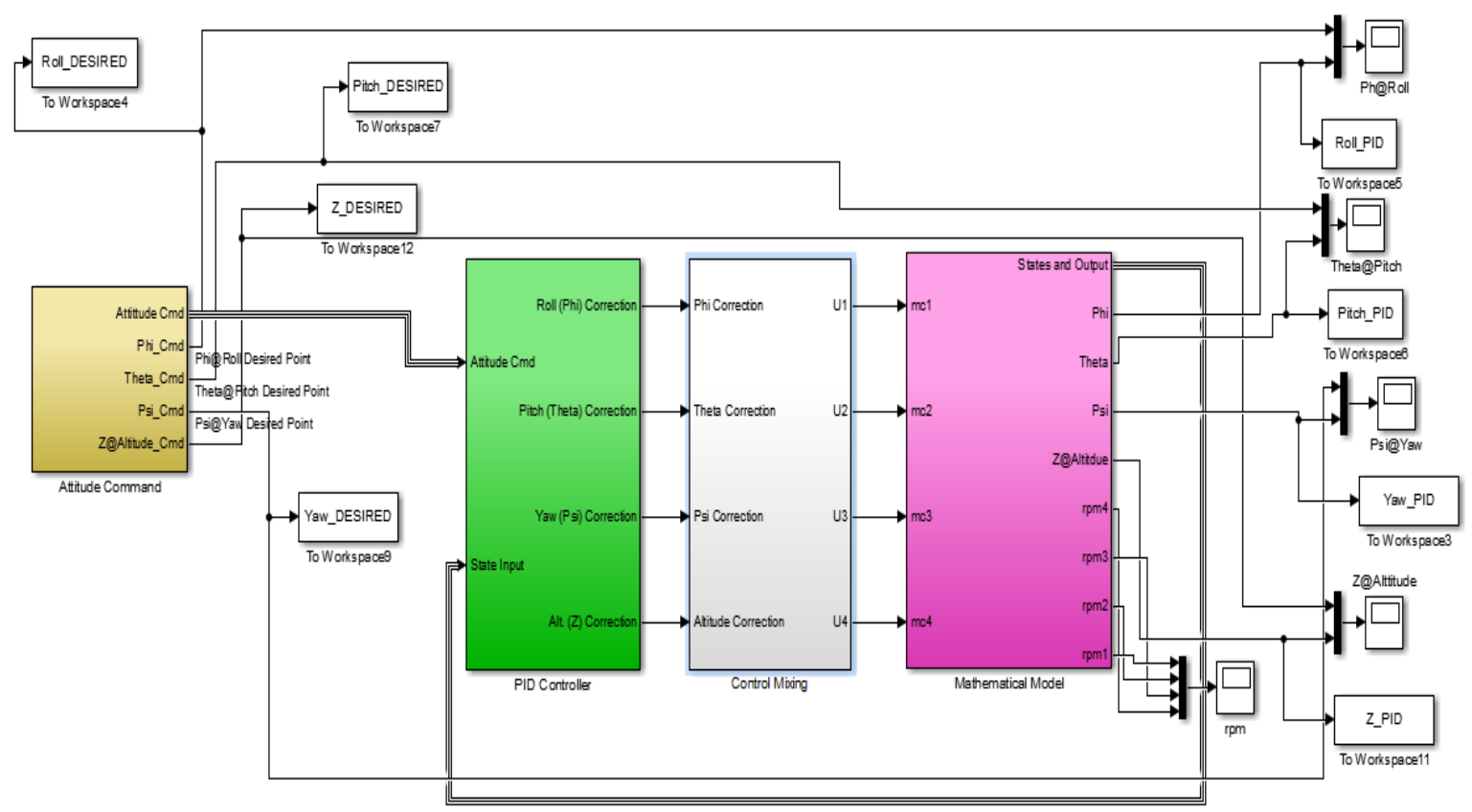

Figure 6. Screenshot of the system implemented in simulation

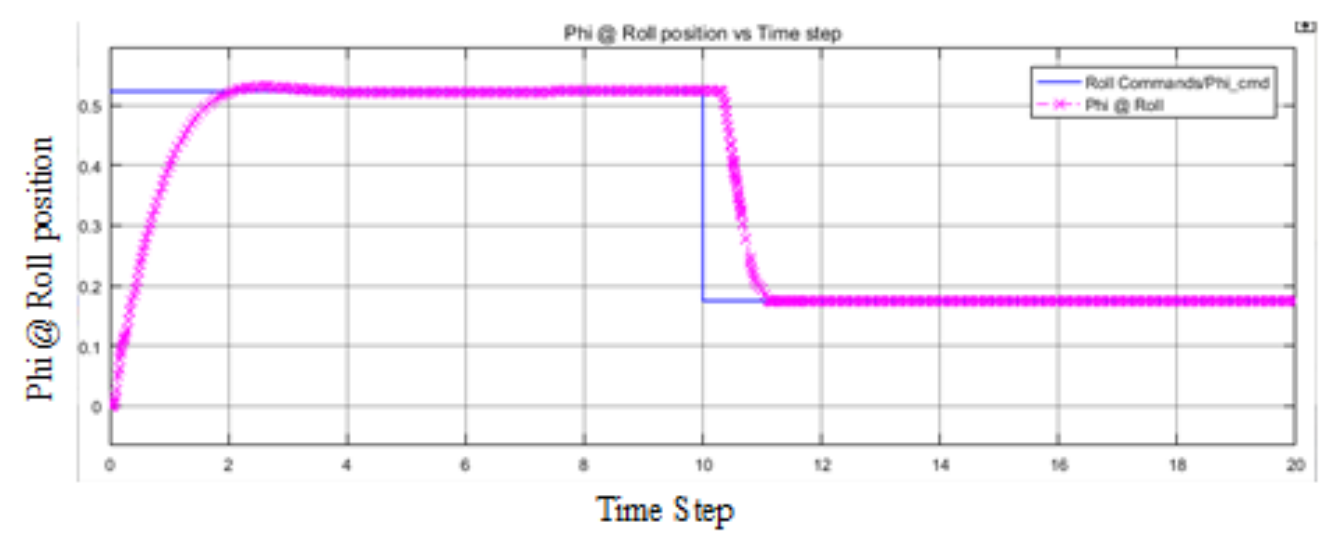

Figure 7. PID block for phi @ roll position

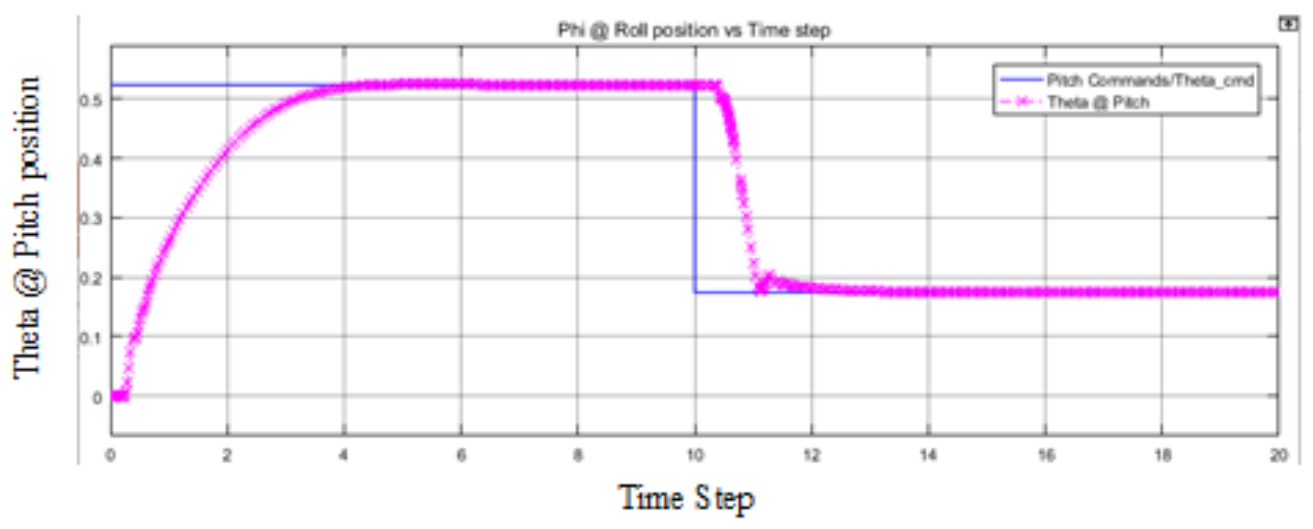

Figure 8. PID block for theta @ pitch position 


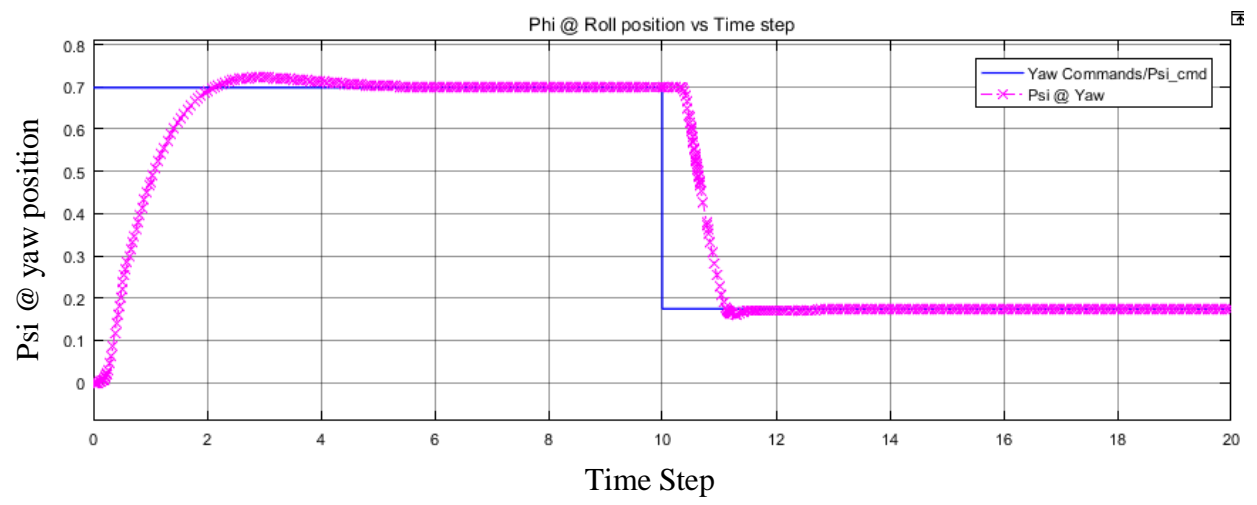

Figure 9. PID block for psi @ yaw position

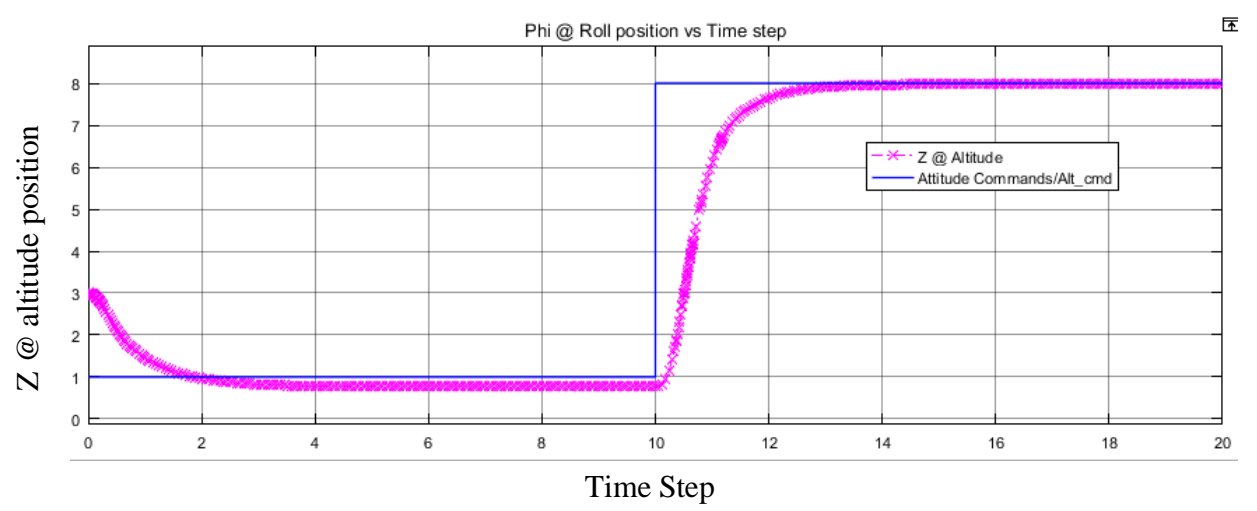

Figure 10. PID block for Z @ altitude position

In the Figure 6 until Figure 9, the straight line is the desired command for the quadcopter to maneuver, while the cluttered $\mathrm{x}$ symbol is the actual quadcopter maneuver output. The time taken for the quadcopter to achieve the desired point, may be varied with the capability of the motors used.

\section{CONCLUSION}

In this paper, the objective of modeling and PID control system integration for quadcopter modelled DJI F450 is achieved by construct the experiment in the simulation using the physical parameter used. The PID increase the stabilization for the DJI F450 efficiently. The development of mathematical modelling of the quadcopter modelled DJI F450 was also presented. For stabilization of the quadcopter, PID controller was chosen, and implemented successfully on MATLAB. The working and performance of quadcopter is tested and desired outputs were obtained using PID controller.

\section{ACKNOWLEDGEMENTS}

The authors would like to thanks Universiti Teknikal Malaysia Melaka (UTeM) under Short Term Research Grant No. PJP/2018/FKEKK(10B)/S01623, Faculty of Electronic and Computer Engineering (FKEKK) and Centre for Telecommunication Research and Innovation (CeTRI) for their support.

\section{REFERENCES}

[1] D. Biediger, et al., "Investigating the survivability of drone swarms with flocking and swarming flight patterns using Virtual Reality," in 2019 IEEE 15th International Conference on Automation Science and Engineering (CASE), pp. 1718-1723, 2019.

[2] K. Loayza, et al., "A centralized control of movements using a collision avoidance algorithm for a swarm of autonomous agents," in 2017 IEEE Second Ecuador Technical Chapters Meeting (ETCM), pp. 1-6, 2017. 
[3] D. Palossi, et al., "Target following on nano-scale unmanned aerial vehicles," in 2017 7th IEEE international workshop on advances in sensors and interfaces (IWASI), pp. 170-175, 2017.

[4] K. Koszela, et al., "Design of an effective platform for unmanned aerial vehicles to collect research material in the form of aerial photographs," in Tenth International Conference on Digital Image Processing (ICDIP 2018), vol. 10806, p. 1080662, 2018.

[5] C. G. Sărăcin, et al., "Powering aerial surveillance drones," in 2017 10th International Symposium on Advanced Topics in Electrical Engineering (ATEE), pp. 237-240, 2017.

[6] A. Alkamachi and E. Erçelebi, "Modelling and genetic algorithm based-PID control of H-shaped racing quadcopter," Arabian Journal for Science and Engineering, vol. 42, no. 7, pp. 2777-2786, 2017.

[7] A. Alkamachi and E. Ercelebi, "Modelling and control of H-shaped racing quadcopter with tilting propellers," Facta Universitatis, Series: Mechanical Engineering, vol. 15, no. 2, pp. 201-215, 2017.

[8] M. A. Alsharif, et al., "A comparison between advanced model-free PID and model-based LQI attitude control of a quadcopter using asynchronous android flight data," in 2017 25th Mediterranean Conference on Control and Automation (MED), pp. 1023-1028, 2017.

[9] N. X. Mung and S. K. Hong, "Improved altitude control algorithm for quadcopter unmanned aerial vehicles," Applied Sciences, vol. 9, no. 10, p. 2122, 2019.

[10] M. A. Alsharif, et al., "Advanced PID attitude control of a quadcopter using asynchronous android flight data," in 2017 International Conference on Unmanned Aircraft Systems (ICUAS), pp. 1602-1607, 2017.

[11] D. Rotondo, et al., "Robust quasi-lpv model reference ftc of a quadrotor uav subject to actuator faults," International Journal of Applied Mathematics and Computer Science, vol. 25, no. 1, pp. 7-22, 2015.

[12] A. A. A. Alawsi, et al., "Nonlinear estimation of quadcopter states using unscented Kalman filter," Periodicals of Engineering and Natural Sciences, vol. 7, no. 4, pp. 1626-1637, 2019.

[13] F. Gazal, "Optimized Endpoint Delivery via Unmanned Aerial Vehicles," Doctoral dissertation, Minnesota State University, Mankato, 2017.

[14] M. Walid, et al., "Modelling, Identification and Control of a Quadrotor UAV," in 2018 15th International MultiConference on Systems, Signals \& Devices (SSD), pp. 1017-1022, 2018.

[15] Y. Alaiwi and A. Mutlu, "Modelling, Simulation and Implementation of Autonomous Unmanned Quadrotor," Machines Technologies Materials, vol. 12, no. 8, pp. 320-325, 2018.

[16] M. Alabsi and T. Fields, "Flight controller learning based on real-time model estimation of a quadrotor aircraft," Proceedings of the Institution of Mechanical Engineers, Part G: Journal of Aerospace Engineering, pp. 3298-3312, 2019.

[17] M. Guiatni, et al., "Fault Tolerant Control Design for Actuator Loss of Effectiveness in Quadrotor Uavs," in 2019 International Russian Automation Conference (RusAutoCon), pp. 1-7, 2019.

[18] M. S. M. Ashraf, et al., "Modelling and Parameters Identification of a Quadrotor Using a Custom Test Rig," International Journal of Power Electronics and Drive Systems (IJPEDS), vol. 9, no. 2, pp. 865-872, 2018.

[19] S. Badr, et al., "A design modification for a quadrotor UAV: modeling, control and implementation," Advanced Robotics, vol. 33, no. 1, pp. 13-32, 2019.

[20] O. I. D. Bashi, et al., "Unmanned aerial vehicle quadcopter: A review," Journal of Computational and Theoretical Nanoscience, vol. 14, no. 12, pp. 5663-5675, 2017.

[21] S. Musa, "Techniques for quadcopter modeling and design: A review," Journal of Unmanned System Technology, vol. 5, no. 3, pp. 66-75, 2017.

[22] A. J. M. Tamayo, et al., "Multirotor modelling and simulation: Screws, SOA, Euler angles, quaternions, wind," in 2017 14th International Conference on Electrical Engineering, Computing Science and Automatic Control (CCE), pp. 1-6, 2017.

[23] H. Xiu, et al., "Dynamic modelling and simulation of a deployable quadrotor," in 2018 International Conference on Reconfigurable Mechanisms and Robots (ReMAR), pp. 1-8, 2018.

[24] I. Al-Ali, et al., "State of the art in tilt-quadrotors, modelling, control and fault recovery," Proceedings of the Institution of Mechanical Engineers, Part C: Journal of Mechanical Engineering Science, pp. 474-486, 2020.

[25] N. T. Hegde, et al., "Modelling and Transition flight control of Vertical Take-Off and Landing unmanned TriTilting Rotor Aerial Vehicle," in 2019 3rd International conference on Electronics, Communication and Aerospace Technology (ICECA), pp. 590-594, 2019.

\section{BIOGRAPHIES OF AUTHORS}

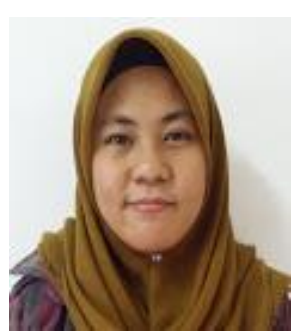

Nur Salma Mohd Mokhtar received B. Eng. (Electronics and Computer) from Universiti Teknikal Malaysia Melaka in 2009 and she is currently pursuing M. Eng. (Electronics and Computer) from Universiti Teknikal Malaysia Melaka. 


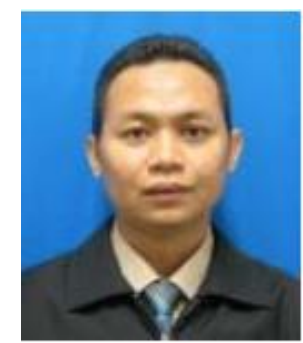

Dr. Khairuddin Osman received B. Eng. in Electronics Engineering (Industrial Electronics) from Universiti Teknikal Malaysia and M. Eng. in Electrical Engineering (Electrical Mechatronics and Automatic Control) from Universiti Teknologi Malaysia. In 2014, he completed Ph.D. degree in Electrical Engineering from Universiti Teknologi Malaysia. He is currently a senior lecturer at the Faculty of Electronics and Computer Engineering, Universiti Teknikal Malaysia Melaka, Malaysia. His interests are in mechatronics, embedded system, pneumatic actuator, industrial electronics, and robotics. 